\section{Indications for and outcome of blood transfusion in dogs in a multi disciplinary referral practice}

\section{Ragnhild Skulberg, Stephen J Baines, Jane Eastwood}

Willows Veterinary Centre and Referral Service, Solihull, West Midlands, UK

\section{INTRODUCTION}

Canine blood products are more available since the 2007 launch of Pet Blood Bank UK. Increased awareness of benefits is balanced against costs and risks involved in transfusion. The decision to transfuse made by clinicians is based on patient assessment, influenced by personal experience.

\section{OBJECTIVES}

Report indications for and outcomes of blood product transfusion.

\section{ANIMALS \\ 151 client-owned dogs.}

\section{METHODS}

Retrospective case series of dogs receiving blood products, August 2009-November 2013. Indications for transfusion and outcome (survival vs non-survival) were recorded.

\section{RESULTS}

151 dogs received transfusions: $29 \mathrm{ME}, 44 \mathrm{MN}, 17 \mathrm{FE}$, and 61
FN. Median age was 78 months (3-183). Common breeds were Labrador (18 dogs), Cocker Spaniel (14 dogs), Springer Spaniel (8 dogs), Jack Russell Terrier (7 dogs) with 87 dogs in other breeds and 17 crossbreeds. Indications were medical diseases (133 dogs) including immune mediated (75 dogs), gastrointestinal (20 dogs), neoplastic (17 dogs), septic (5 dogs), coagulopathic ( 8 dogs), and nasal disease ( 4 dogs), haemoabdomen ( 2 dogs), trauma (2 dogs), and surgical blood loss (18 dogs).

Transfusions included PRBCs (154 dogs), whole blood (50 dogs), FFP (13 dogs), cryoprecipitate (3 dogs), and autotransfusion (1 dog). Recipient blood type was known in 90 dogs: 1.1-ve (42 dogs) and 1.1+ve (48 dogs). Crossmatching was performed in 24 dogs. Number of transfusions was: one (103 dogs), two (34 dogs), three (8 dogs), four (4 dogs), or five (2 dogs). Median PCV (\%)/TP (g/dl) was 15/52 pre-transfusion and 25/56 post-transfusion. Median absolute increase in PCV (\%)/TP $(\mathrm{g} / \mathrm{dl})$ was $10 / 2$. No major transfusion reactions were reported.

Median hospitalisation was 3 days (1-15) overall and 2 days (1-11) post-transfusion. 117 (77.5\%) dogs were discharged at a median of 3 days (1-15). Causes of death were cardio-pulmonary arrest (11 dogs), clinical deterioration (8 dogs) and euthanasia at owners request (15 dogs). There was no difference between dogs with medical diseases or surgical blood loss in survival rate $(78.2 \%$ vs $72.2 \%, P=0.556)$, cause of death $(P=0.300)$ or blood product received $(P=0.230)$, but there were significant differences in PCV/TS pre- and posttransfusion $(P<0.001-0.0076)$. Survival was not associated with number of transfusions $(P=0.525)$ or type of medical disease $(P=0.181)$, but was associated with $P C V$ post-transfusion $(P=0.014)$

\section{CONCLUSIONS AND CLINICAL IMPORTANCE}

$77.5 \%$ dogs survived, supporting use of blood products in veterinary practice. Non survival was due to primary disease not transfusion complications.

\section{Evaluation of red blood cell distribution width in canine septic peritonitis}

\section{Alexander Forward, Giacomo Stanzani, Stefano Cortellini, Lindsay Kellett- Gregory}

Royal Veterinary College, London, UK

\section{BACKGROUND}

Canine septic peritonitis is a challenging condition with a reported mortality rate range of $27-80 \%$. Prognostic factors for such conditions enable owners to make informed decisions with regard to continuing care and also help to heighten clinicians' awareness of critically ill patients. Red blood cell distribution width (RDW), a quantitative measure reflecting the variability in size of circulating erythrocytes that is routinely reported as part of complete blood counts (CBC), has recently been suggested to be a prognostic indicator in a number of human conditions, including sepsis.

\section{OBJECTIVE}

To investigate the association of RDW and other laboratory variables with all cause mortality in dogs with septic peritonitis.

\section{DESIGN}

Retrospective, study conducted at a teaching hospital.

\section{ANIMALS}

Sixty client-owned dogs with septic peritonitis

\section{METHODS}

Electronic records were searched and analysed for cases that underwent surgery for the treatment of septic peritonitis in the 